Disclosure of Interests: None declared

DOI: 10.1136/annrheumdis-2019-eular.8498

\section{SP0026 $\quad$ METHODS AND TOOLS FOR QUANTIZATION OF CAPILLAROSCOPIC MORPHOLOGICAL CHANGES}

Alberto Sulli. University of Genoa, Research Laboratory and Academic Division of Clinical Rheumatology, Department of Internal Medicine, IRCCS Polyclinic Hospital San Martino, Genova, Italy

Capillaroscopy is routinely used in clinical practice to assess nailfold microvascular structure. Unlike laser techniques that give functional information, capillaro scopy allows a morphological assessment of nailfold capillary bed. It is a recognized tool to early differentiate primary from secondary Raynaud's phenomenon, as well as to follow scleroderma microangiopathy. As microangiopathy extent correlates with organ involvement degree, nailfold capillaroscopy abnormalities are also considered a possible biomarker in systemic sclerosis. ${ }^{1,2}$

Several tools may be used to quantitate morphological capillary changes, from devices with low magnification (20x), to instruments with high magnification (200x or more). ${ }^{3}$ The instruments with low magnification allow a global evaluation of the entire nailfold area (wide-field capillaroscopy), and they provide a panoramic vision of the whole nailfold microvascular network. Magnifying glass, ophthalmoscope and dermatoscope are example of optical instruments with low magnification. Obtaining a high-quality documentation may be difficult by these tools, as well as the identification of early capillary changes; furthermore, storing capillary images is not possible.

Stereomicroscope, usb-microscope or videocapillaroscope allow a sequential higher magnification up to $600 x$ which enable detailed observation of small nailfold areas or single capillaries. However, by using a microscope, patient's fingers must be placed under the lens, and this may make the exam sometimes difficult, for example in the presence of either finger contractures or arm disabilities.

The optical probe videocapillaroscope is currently considered the gold-standard for nailfold videocapillaroscopy, as it has the advantage to be moved to the finger of the patient at direct contact with the nailfold: this facilitates examination of patients with systemic sclerosis and finger flexion contractures, simply by moving the probe. The use of videocapillaroscope has a high sensitivity, a short time of execution after an easy operator training, allows early diagnosis of secondary Raynaud's phenomenon by very early detection of microvascular changes, and also allows the counting and scoring of capillaroscopic parameters. Connection of the instrument with a software enable image recording, report writing and automated capillary number counting. ${ }^{4}$

To quantitate capillaroscopic morphological changes (mainly capillary number, capillary dilations, giant capillaries, microhemorrhages, abnormal shape/ramified capillaries, capillary bed disorganization) a simple rating scale may be adopted to score each capillary abnormalities (score $0-3$ ) (score $0=$ no changes; score $1=$ less than $33 \%$ of capillary alterations/reduction; score $2=33-66 \%$ of capillary alterations/reduction; score $3=$ more than $67 \%$ of capillary alterations/reduction, per linear millimetre. Reduction = capillary number per linear millimetre $<9) .{ }^{5}$ The mean score value for each capillaroscopic parameter is calculated from the analysis of at least two millimetres in the centre of the nailfold, by evaluating the first row of capillaries in each finger from $2^{\text {nd }}$ to $5^{\text {th }}$.

The Microangiopathy Evolution Score (MES) (sum of scores of progression parameters: capillary number, abnormal shaped/ramified capillaries, capillary bed disorganization - Score 0-9) may be calculated to follow the scleroderma microvascular damage. ${ }^{5}$ However, at least the simple capillary number per linear millimetre should be counted, as well as larger capillary diameters measured, to monitor microangiopathy in systemic sclerosis. ${ }^{4,6,7}$

\section{REFERENCES:}

[1] Smith V, et al. J Rheumatol 2013;40:2023-8

[2] Ingegnoli F, et al. Microvasc Res 2013;89:122-8.

[3] Cutolo M, et al. Best Pract Res Clin Rheumatol 2013;27:237-48.

[4] Cutolo M, et al. Microcirculation 2018;25:e12447.

[5] Sulli A, et al. Ann rheum Dis 2008;67:885-7.

[6] Cutolo M, et al. Arthritis Rheumatol 2016;68:2527-39.

[7] Trombetta AC, et al. J Rheumatol 2016;43:599-606.

Disclosure of Interests: None declared

DOI: 10.1136/annrheumdis-2019-eular.8597
WEDNESDAY, 12 JUNE 2019

$16: 15: 00-17: 45: 00$

\section{Bringing digital health care solutions to patients}

\section{SP0027 \\ E-HEALTH REDEFINES THE RELATIONSHIP BETWEEN PATIENTS WITH RMDS AND HEALTH CARE} PROFESSIONALS

Paul Studenic. Medical University of Vienna, Internal Medicine 3, Division of Rheumatology, Vienna, Austria

By definition of the World Health Organization (WHO), e-health relates to the use of information and communication technologies for health services. This incorporates digital patient charts, access to their respective health records by the patient and treating physicians as well as medical apps (applications) for monitoring of health parameters and interacting with health care professionals and peers. The use and initiatives and legislations concerning e-health have become increasingly wide spread across the world. With the continued popularity of smartphones in recent years, both smartphone applications and social media are progressively used. In 2018, worldwide users of social media were estimated at 3.196 billion, accounting for $42 \%$ of the global population. Social media use has grown enor mously in the last decade, with a plus of $13 \%$ in 2017 alone. More than half and two thirds of the world's population are connected to the internet and having a mobile phone respectively. The roll-out of mobile solutions, affordable smartphones and broadband internet in remote areas on the globe allow for quicker and more specialised medical encounters. These developments enable people also to conveniently interact, access knowledge and take part in continued medical education regardless of geographical location. Mobile health (m-health) services range from toll free emergency numbers or health call centres (available in $75 \%$ of WHO countries) to treatment adherence and surveillance programs $(48 \%$ of $\mathrm{WHO}$ countries). Fields, like radiology apply telehealth already very successfully, which leads to greater health equity globally. Therefore, e-health provides people with health conditions, particularly chronic conditions such as rheumatic and musculoskeletal diseases (RMDs), with new opportunities to identify information about their disease and treatment, as well as the ability to connect.

The use of e-health solutions for collecting patient reported outcomes (PRO) enables patients to more realistically depict on how they are doing over time, provides better comparisons and changes the dynamic of feedback and the potential most appropriate time point for clinical encounters. Online health users with rheumatoid arthritis do currently however not represent the general population of people with $\mathrm{RA}$, since there are more often younger, do have shorter disease duration and live in rural areas. At the same time, they define an additional group for clinical trials and have a high willingness to participate, which outlines new avenues for recruitment of patients into trials. Still, continued constant use of $\mathrm{m}$-health applications is less common. One third of people that started using a mobile health app are using it on daily or weekly basis. The use of apps does not always coincide with bette outcomes as could be shown in a trial incorporating people with poorly controlled hypertension. This indicates, despite all positive developments and opportunities over the last decade, that there is still a long way ahead to tailor e-health initiatives to result in more optimised work flows for health care professionals and better health management for everyone.

Disclosure of Interests: None declared

DOI: 10.1136/annrheumdis-2019-eular.8596

\section{SP0028 RMD PATIENTS BE LIKE?}

Aurelie Najm. Nantes University Hospital, Rheumatology, Nantes, France

Mobile health (mhealth) connects patients, their families and physicians by creat ing a network with mobile and specialized devices such as wearable sensors, recording health parameters and gathering health data converted and transferred to physicians via medical application interfaces. Apps for people with chronic health conditions have an important role to play in empowering those individuals to become more active in the self- and shared-management of their health. Since rheumatic and musculoskeletal diseases (RMDs) have multi-dimensional consequences on health, the increasing availability of apps have an important role to play in enabling people with RMDs to better self-manage their health. This talk provides insights into the most important aspects to be considered for apps designed for people living with RMDs: features, use, safety, and patient's views and preferences.

Disclosure of Interests: None declared

DOI: 10.1136/annrheumdis-2019-eular.8545 